Research Paper

\title{
Long non-coding RNA RP4-694A7.2 Promotes Hepatocellular Carcinoma Cell Proliferation and Metastasis through the Regulation of PSAT1
}

\author{
Yaoxin Fan 1,2, Lin Wang3, Yang Ding1,2, Qiuju Sheng1,2, Chong Zhang1,2, Yanwei Li1,2, Chao Han ${ }^{1,2}$, \\ Bingchao Lu ${ }^{1,2}$ and Xiaoguang Dou ${ }^{1,2}$ \\ 1. Department of Infectious Diseases, Shengiing Hospital of China Medical University, No. 39, Huaxiang Road, Shenyang Liaoning province, China \\ 2. Key Laboratory of Viral hepatitis, Shengjing Hospital of China Medical University, No. 39, Huaxiang Road, Shenyang Liaoning province, China \\ 3. Department of Health Management, Shengjing Hospital of China Medical University, No. 39, Huaxiang Road, Shenyang Liaoning province, China \\ $\triangle$ Corresponding author: Xiaoguang Dou. Address: Department of infectious diseases, Shengjing hospital of China medical university, No. 39, Huaxiang Road,
} Shenyang Liaoning province, China; Tel: +86-18940251121.E-mail: douxg@sj-hospital.org

(c) The author(s). This is an open access article distributed under the terms of the Creative Commons Attribution License (https://creativecommons.org/licenses/by/4.0/). See http://ivyspring.com/terms for full terms and conditions.

Received: 2021.02.12; Accepted: 2021.07.10; Published: 2021.07.25

\begin{abstract}
Background: Long noncoding RNAs (IncRNAs) have emerged as gene regulators in various cancers, including hepatocellular carcinoma (HCC). However, the biological roles and mechanisms of many IncRNAs in HCC tumorigenesis remain unknown.

Aim: To identify novel IncRNAs associated with proliferation and metastasis in HCC.

Methods: Expression profiles of IncRNAs were analyzed in HCC using two GSE datasets (GSE94660 and GSE104310). Functional studies were performed, including cell proliferation, colony formation, wound healing, and Transwell assays. Fluorescence in-situ hybridization (FISH), tandem mass tag (TMT) analyses, parallel reaction monitoring (PRM), and rescue assays were performed to evaluate the mechanisms underlying the effects of RP4-694A7.2.

Results: RP4-694A7.2 levels were higher in HCC tissues than in normal liver tissues in published GSE datasets and were elevated in HCC cell lines. Cell function assays revealed that RP4-694A7.2 promotes cell proliferation, invasion, and migration. Furthermore, RP4-694A7.2 was primarily found to be located in the cytoplasm by FISH assay. Then, TMT assay was performed to predict proteins associated with RP4-694A7.2, and 28 cytoplastic proteins were identified by PRM. Finally, phosphoserine aminotransferase 1 (PSAT1) was found to be regulated by RP4-694A7.2 to modulate growth and metastasis in HCC cells using a rescue assay.

Conclusions: These results suggested that RP4-694A7.2 promotes HCC cell proliferation and metastasis via PSAT1, providing a candidate therapeutic target for further research.
\end{abstract}

Key words: lncRNA RP4-694A7.2, cell proliferation, invasion and migration, hepatocellular carcinoma

\section{Introduction}

Hepatocellular carcinoma (HCC) is a leading cause of cancer-related deaths worldwide. Despite treatment advances, HCC mortality is still high [1,2]. The lack of therapeutic strategies can be explained by the limited understanding of the mechanisms underlying HCC, which are complex diverse and vary among individuals [3]. Therefore, studies of the underlying mechanism are expected to improve outcomes and decrease mortality in HCC.
Long non-coding RNAs (lncRNAs) are a class of non-coding RNAs more than 200 nucleotides in length [4]. IncRNAs regulate gene expression at the epigenetic, transcriptional, and post-transcriptional levels and can promote or suppress cell signaling pathways in different types of cancer $[5,6]$. Accumulating evidence has revealed the importance of IncRNAs in the development of HCC, including roles in proliferation, angiogenesis, metastasis, and 
genomic stability [7-13]. Although several lncRNAs involved in the development of HCC have been identified, the precise functions of most lncRNAs have not been characterized. For example, lncRNA RP4-694A7.2 was previously described to be upregulated in some solid cancers compared to their respective normal tissues through bioinformatics analyses [14]. This lncRNA was also overexpressed in highly proliferative stages of normal B cell differentiation [15]. Although those studies showed that RP4-694A7.2 was associated with cancers, the function of the molecule in most cancers remains unclear.

In the present study, differentially expressed lncRNAs in two HCC-related GSE datasets (GSE94660 and GSE104310) were analyzed. The expression and localization of the candidate lncRNA RP4-694A7.2 were further assessed in HCC cells. Functional experiments suggested that RP4-694A7.2 promotes proliferation and metastasis in HCC. Phosphoserine aminotransferase 1 (PSAT1), an important factor in HCC, was regulated by PR4-694A7.2, as determined by tandem mass tags and parallel reaction monitoring. Our study suggested that RP4-694A7.2 could be a potential therapeutic target in HCC.

\section{Materials and Methods}

\section{GEO data}

GEO (Gene Expression Omnibus, http://www .ncbi.nlm.nih.gov/geo) is an international public database of microarray data, second-generation sequencing data, and other high-throughput sequencing data [16]. Two GSE (series) HCC datasets (GSE94660 and GSE104310) were downloaded from the GEO.

\section{IncRNA expression profile analysis}

lncRNA-Seq high-throughput sequencing and subsequent bioinformatics analyses were all performed by Cloud-Seq Biotech (Shanghai, China). Briefly, paired-end reads were subjected to a quality control threshold of Q30. After 3' adaptor-trimming and low-quality read removal using cutadapt (v. 1.9.3), the high-quality trimmed reads were aligned to the human reference genome (UCSC hg19) using HISAT2 (v. 2.0.4). Next, HTSeq (v. 0.9.1) was used to obtain raw counts at the gene level (mRNA) and transcript level (lncRNA) as the expression profiles, and edgeR (v. 3.16.5) was used to normalize the counts and screen differentially expressed lncRNAs and mRNAs.

\section{Cell culture}

$\mathrm{LO}_{2}$ and SK-Hep1cells were purchased from the Cell Bank, Type Culture Collection, Chinese Academy of Sciences (CBTCCCAS). All cells were incubated under standard culture conditions $\left(37^{\circ} \mathrm{C}\right.$ and $\left.5 \% \mathrm{CO}_{2}\right)$.

\section{Quantitative real-time PCR (RT-PCR)}

Total RNA was extracted from cells using TRIzol (Invitrogen, Carlsbad, CA, USA) following the manufacturer's instructions. Real-time PCR was conducted using the SYBR Green PCR Kit (Takara Biotechnology, Dalian, China) and the LightCycler 480 system following the manufacturer's instructions. GAPDH was used as a control. Relative expression levels were calculated using the $2^{-\Delta \Delta \mathrm{Ct}}$ method. All primers are described in Table 1.

Table 1. Primers used in IncRNA screening

\begin{tabular}{|c|c|c|}
\hline Gene Name & & Sequence \\
\hline \multirow[t]{2}{*}{ CTB-147C22.8 } & -forward & 5'- CTCAGGTAGAGAGGAACCACAAGG-3' \\
\hline & - reverse & 5'- AGGCAGGGAAGGAGAGGTGT -3' \\
\hline \multirow[t]{2}{*}{ RP4-694A7.2 } & -forward & 5'- GTGATGTAGCCACAAACAACTACCA -3' \\
\hline & - reverse & 5'- GGGAAAACCACTCATCTGTCTTG -3' \\
\hline \multirow[t]{2}{*}{ RP11-428C19.4 } & -forward & 5'-ACAGTCTGGAATTTACGAGGATGAG -3' \\
\hline & - reverse & 5'-CCTGTGTGTGGTTGACTTCTTTTG -3' \\
\hline \multirow[t]{2}{*}{ HAO2-IT1 } & -forward & 5’-TTTGCCTTCTTGAGGATGGAG-3' \\
\hline & - reverse & 5'-GGAGTGGGAATAGCAGGTGTG -3' \\
\hline \multirow[t]{2}{*}{ HOXD-AS2 } & -forward & 5'-CATAGCCATGCAGCCTTCAG-3' \\
\hline & - reverse & 5'-AGTGCTATTTCACATCCAAGCTTCT-3' \\
\hline \multirow[t]{2}{*}{ HOXA10-AS } & -forward & 5'-CCGCAAATGCGTCAAGGA-3' \\
\hline & - reverse & 5'-AAGGGCTTACAGGCATGGACA-3' \\
\hline \multirow[t]{2}{*}{ GAPDH } & -forward & 5'-GCACCGTCAAGGCTGAGAAC-3' \\
\hline & - reverse & 5'-TGGTGAAGACGCCAGTGGA-3' \\
\hline
\end{tabular}

\section{LV-RP4-694A7.2-RNAi construction}

For RP4-694A7.2 knockdown, interfering lncRNAs targeting RP4-694A7.2 were acquired from GeneChem (Shanghai, China). To construct LV-RP4-694A7.2-RNAi, SK-Hep1 cells suspended in DMEM were incubated in a 6-well plate. Next, cells were incubated with the lentivirus packaging vector (GV112). Fluorescence was captured using a fluorescence microscope (Olympus, Tokyo, Japan) at $72 \mathrm{~h}$ after lentiviral infection. The knockdown efficiency was examined by RT-PCR.

\section{Cell proliferation assay}

SK-Hep1 cells were incubated in 96-well plates (2000 cells per well) under standard culture conditions. Cell viability was measured using Cell Counting Kit-8 (CCK-8; Dojindo, Kumamoto, Japan). The plates were detected using a Microplate Reader (Tecan Infinite, M2009PR) at $450 \mathrm{~nm}$ after $4 \mathrm{~h}$ of incubation

\section{Colony formation assay}

After transfection for $72 \mathrm{~h}$, SK-Hep1 cells were incubated in 6-well plates (1000 cells per well). After culture for 2 weeks, colonies were dyed with Crystal Violet Staining Solution and evaluated. 


\section{Wound-healing assay}

Suspended SK-Hep1 cells were added to a 96-well plate at $5 \times 10^{5} / \mathrm{mL}$ per well. A scratch tester was used to draw lines on the plates. Next, samples were washed with serum-free medium and added to serum-based medium. Images were obtained at 0, 24, and $32 \mathrm{~h}$. Cell mobility (\%) was calculated according to the following formula: $(1$ - scratch width/initial scratch width) $\times 100$.

\section{Transwell assay}

SK-Hep1 cells were suspended and incubated in 24 -well plates $\left(5 \times 10^{5}\right.$ cells per well). In the upper chamber, $100 \mu \mathrm{L}$ of cell suspension was added. In the lower chamber, $600 \mu \mathrm{L}$ of FBS culture medium was added. After $16 \mathrm{~h}$ of culture, the chambers were stained with Crystal Violet Staining Solution and evaluated.

\section{Fluorescence in-situ hybridization (FISH)}

SK-Hep1 cells were fixed in paraformaldehyde (DEPC) for $20 \mathrm{~min}$ and washed three times with PBS. Proteinase $\mathrm{K}(20 \mu \mathrm{g} / \mathrm{mL})$ was added to cover tissues, followed by incubation at $37^{\circ} \mathrm{C}$ for $8 \mathrm{~min}$. Hybridization buffer was added to the specimen, followed by incubation at $37^{\circ} \mathrm{C}$ for $1 \mathrm{~h}$. The probe hybridization solution was added and incubated in a humidity-controlled chamber for hybridization at $37^{\circ} \mathrm{C}$ overnight. Samples were incubated with DAPI for $8 \mathrm{~min}$ in the dark and mounted. Images were obtained using a positive fluorescence microscope.

\section{Tandem mass tag (TMT)}

After samples were digested, they were labeled using TMT reagent according to the manufacturer's instructions (Thermo Fisher Scientific, Waltham, MA, USA). TMT-labeled peptides were fractionated by RP chromatography using the Agilent 1260 infinity II HPLC and evaluated by LC-MS/MS. A peptide and protein false discovery rate of $1 \%$ was enforced using a reverse database search strategy [17]. Proteins with fold change $>1.2$ and $p$-value (Student's $t$-test) $<0.05$ were considered differentially expressed.

\section{Parallel reaction monitoring (PRM) analysis}

To verify the accuracy of the TMT and LC-MS/MS analyses, PRM protein information was imported into Xcalibur for an analysis using PRM parameter settings. Peptides $(1 \mu \mathrm{g})$ were collected from each sample for LC-PRM/MS, and chromatographic separation was performed using the Easy nLC 1200 chromatography system (Thermo Scientific) with a nanoliter flow rate after loading. The sample was subjected to gradient separation through a chromatography column (Thermo Fisher Scientific; Acclaim PepMap RSLC $50 \mu \mathrm{m} \times 15 \mathrm{~cm}$, nano viper, $\mathrm{P} / \mathrm{N164943).} \mathrm{Peptide} \mathrm{fragmentation} \mathrm{and} \mathrm{targeted}$ PRM mass spectrometry were performed using a Q-Exactive HF-X Mass Spectrometer (Thermo Scientific). Raw data obtained by mass spectrometry were analyzed using SpectroDive for PRM data.

MS raw data were processed using Proteome Discoverer (v. 2.1) and SpectroDive (v. 10.0). In Proteome Discoverer, the derived peak lists were searched using the MASCOT engine (Matrix Science, London, UK; version 2.6) against the UniProt Homo sapiens protein database (UP000005640, 20367 sequences, downloaded on February 26, 2020). In SpectroDive, the MS/MS table output file from MASCOT was used to create a spectral library.

\section{Statistical analysis}

All data were analyzed using SPSS v. 22.0 (SPSS Inc., Chicago, IL, USA). Differences between control and experimental groups were evaluated by Student's $t$-tests. Data are reported as means \pm standard deviation. Values of $p<0.05$ were considered significant.

\section{Results}

\section{RP4-694A7.2 was upregulated in HCC}

To screen unique lncRNAs related to HCC, two GSE datasets (GSE94660 and GSE104310) were downloaded from the GEO for bioinformatics analyses. In total, 1409 lncRNAs were upregulated while 1508 lncRNAs were downregulated in HCC. Heat maps for the GSE94660 and GSE104310 datasets are shown in Figure 1A, and a volcano map of differentially expressed lncRNAs is shown in Figure 1B. In total, 387 lncRNAs were upregulated and 604 were downregulated in GSE104310, while 1022 lncRNAs were upregulated and 904 were downregulated in GSE94660. A Venn diagram was generated to visually evaluate the overlap between lncRNAs. We found 347 upregulated and 147 downregulated lncRNAs (Figure 1C).

We focused on upregulated lncRNAs, which are more suitable than downregulated lncRNAs as diagnostic biomarkers or therapeutic targets [18]. The top 50 upregulated IncRNAs in GSE94600 and GSE104310 were analyzed. RP4-694A7.2 was a highly expression lncRNA and was therefore further evaluated in SK-Hep1 cells by qRT-PCR. Compared with levels in $\mathrm{LO}_{2}$ cells, RP4-694A7.2 was significantly upregulated in SK-Hep1 cells (Figure 1D). Accordingly, RP4-694A7.2 might play a role in HCC. 
A
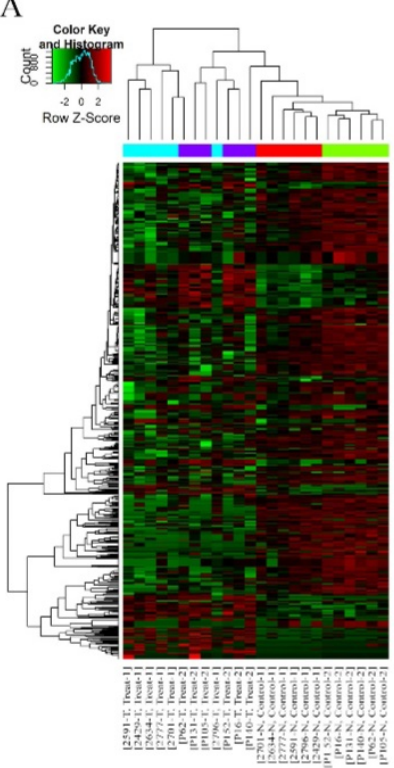

B

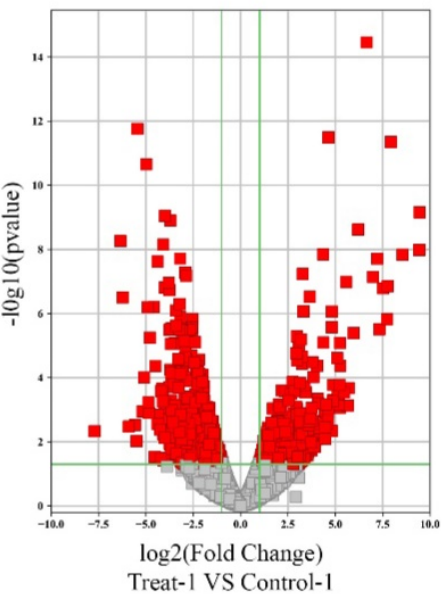

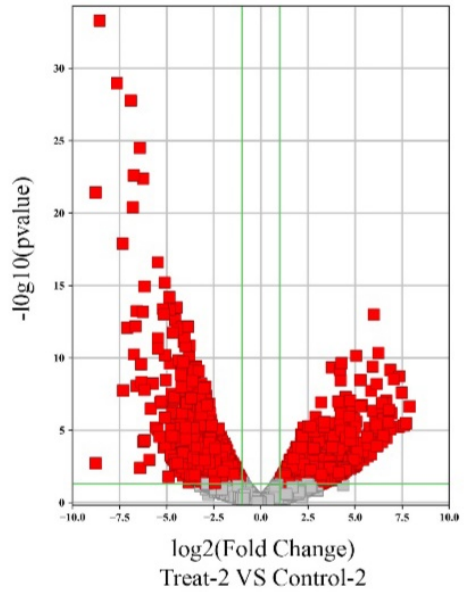

$\log 2$ (Fold Change)
Treat-2 VS Control-2

C
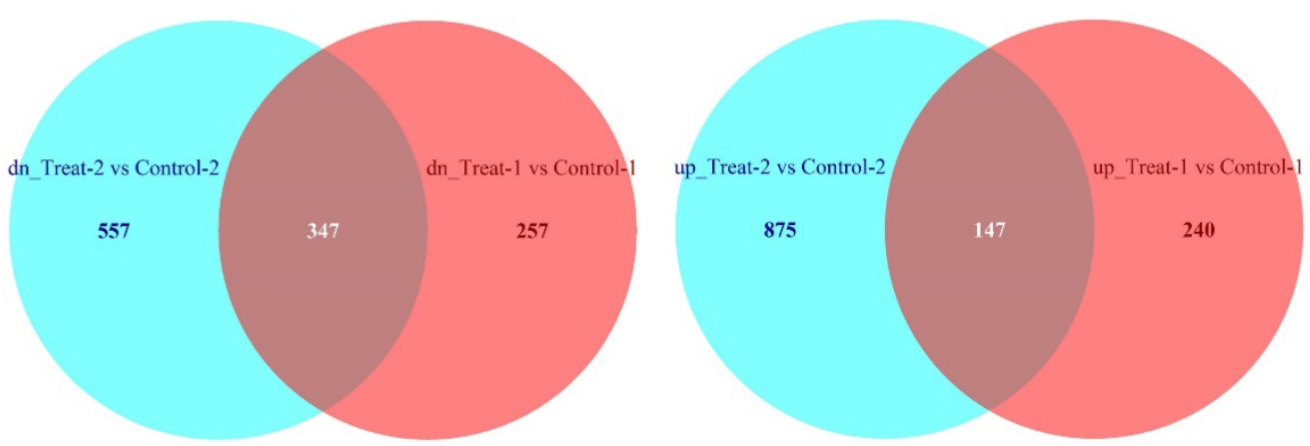

D

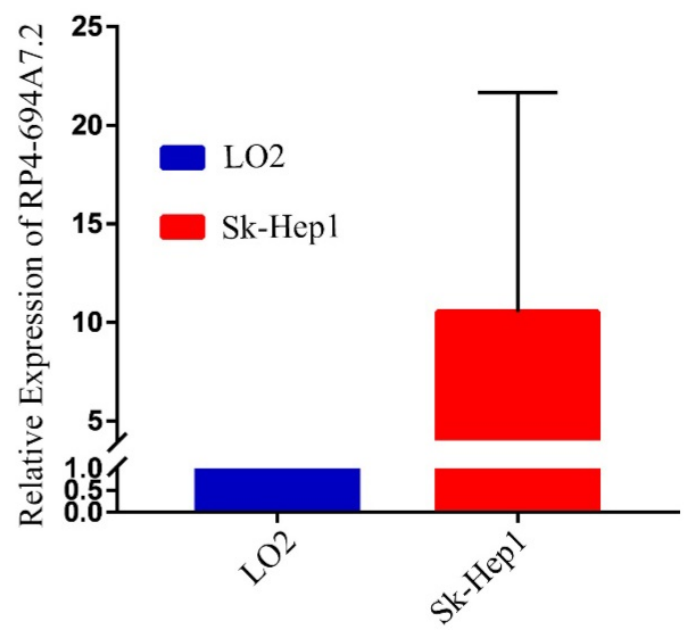

Figure 1. RP4-694A7.2 levels were increased in HCC. A: Dysregulated IncRNAs were screened by a bioinformatics approach using the GSE94660 and GSE104310 datasets. B: Volcano map of differentially expressed IncRNAs. C: Venn diagram showing the intersection between IncRNAs in the GSE94660 and GSE104310 datasets. D: RP4-694A7.2 was examined by qRT-PCR in HCC (SK-Hep-1) and LO 2 cell lines $(p<0.05)$.

\section{Knockdown of RP4-694A7.2 inhibits HCC cell proliferation}

To explore the function of RP4-694A7.2 in HCC proliferation, we knocked down RP4-694A7.2 in
SK-Hep-1 cells and confirmed the knockdown efficiency by qRT-PCR. The RP4-694A7.2 level was markedly decreased in HCC cells after knockdown. According to a CCK-8 assay, RP4-694A7.2 knockdown 
remarkably reduced the rate of cell proliferation compared with that in the control group on days 4 and 5 in SK-Hep-1 cells (Figure 2A). The knockdown of RP4-694A7.2 also inhibited SK-Hep-1 cell proliferation in a colony formation assay (Figure 2B).

\section{Knockdown of RP4-694A7.2 inhibits HCC cell migration and invasion}

We further evaluated the effects of RP4-694A7.2 knockdown on migration and invasion in HCC cells.
According to a wound-healing assay, RP4-694A7.2 knockdown delayed wound healing (Figure 2C), suggesting reduced migration. Based on Transwell migration and invasion assays, the knockdown of RP4-694A7.2 significantly reduced migrating and invading cell counts (Figure 2D).

Taken together, RP4-694A7.2 knockdown could inhibit HCC cell migration and invasion.

A

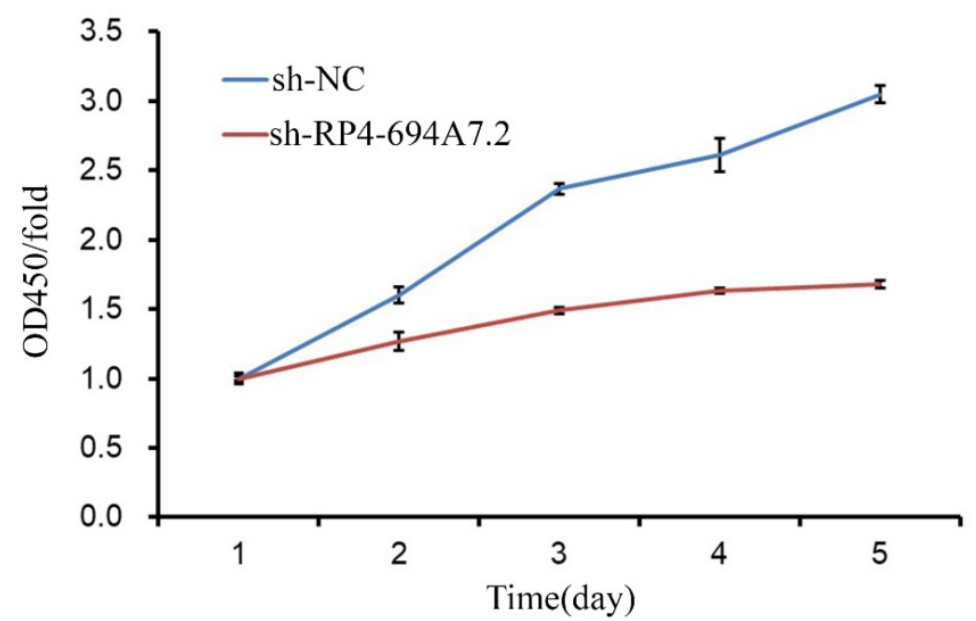

B

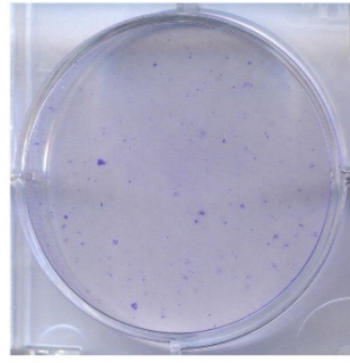

sh-NC

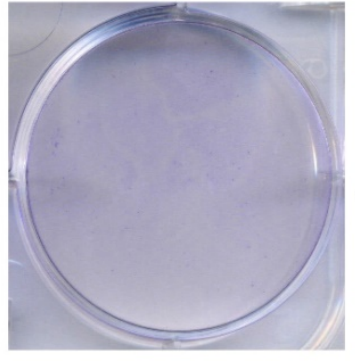

sh-RP4-694A7.2

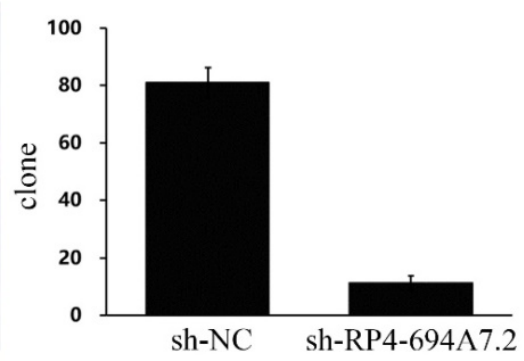

C

$0 \mathrm{~h}$
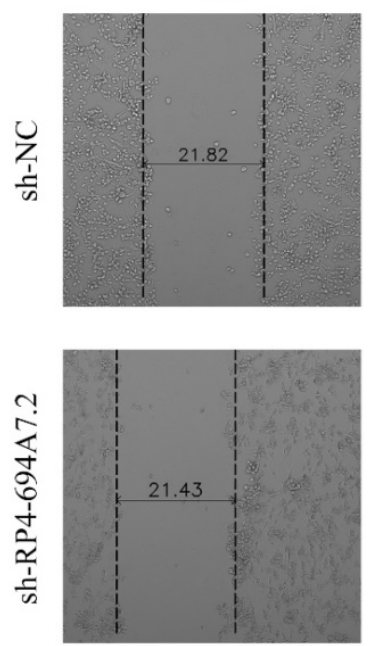

$24 \mathrm{~h}$
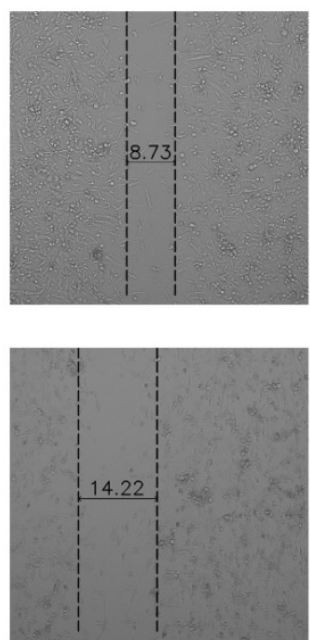

$32 \mathrm{~h}$
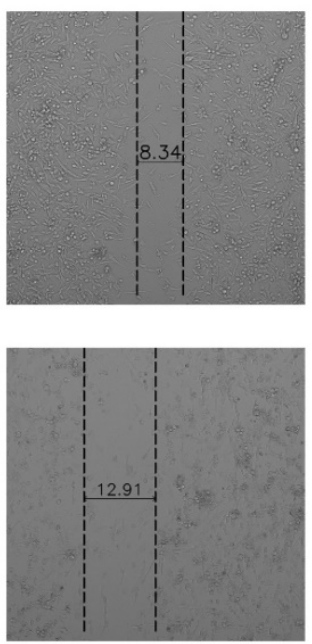
D
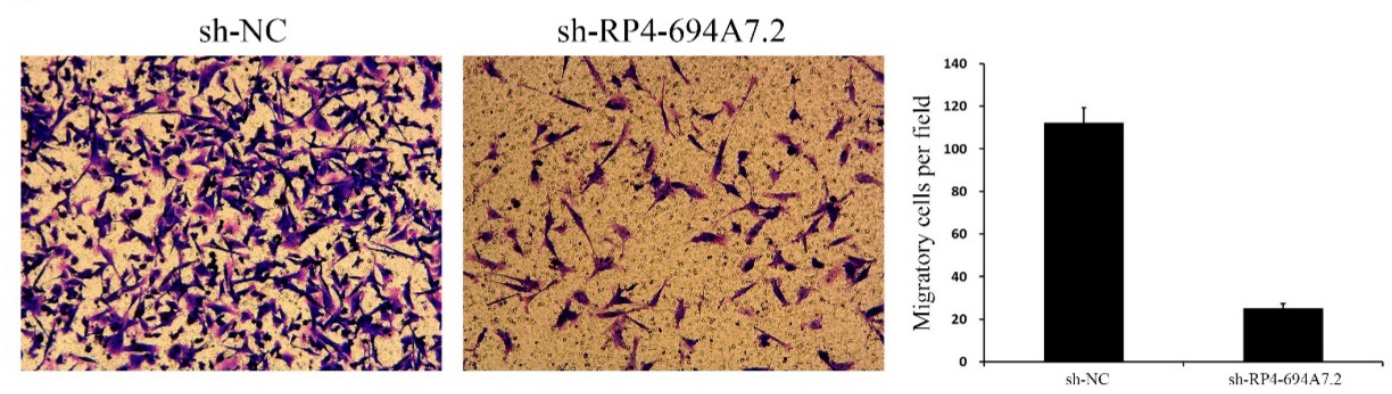

E
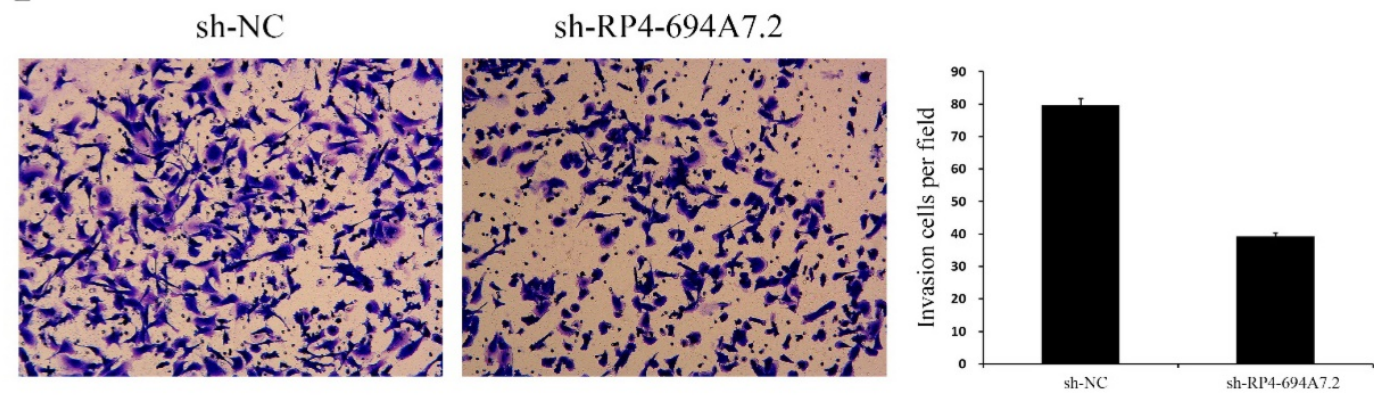

Figure 2. Knockdown of RP4-694A7.2 inhibited HCC cell proliferation, migration, and invasion. A: Knockdown of RP4-694A7.2 in HCC cells inhibited proliferation, as determined by a CCK-8 assay $(p<0.05)$. B: Knockdown of RP4-694A7.2 inhibited the formation of cell clones $(p<0.05)$. C: Knockdown of RP4-694A7.2 inhibited HCC cell migration, as determined by a wound-healing assay. D: Knockdown of RP4-694A7.2 inhibited the migration of HCC cells, as determined by a Transwell assay $(p<0.05)$. E: Knockdown of RP4-694A7.2 inhibited the invasion of HCC cells, as determined by a Transwell assay $(p<0.05)$. Data are expressed as means \pm SD of three independent experiments.

\section{Screening proteins associated with RP4-694A7.2 in HCC cells}

To investigate the molecular mechanism underlying the effects of RP4-694A7.2 in HCC, a TMT assay was performed to predict proteins associated with RP4-694A7.2. The TMT assay results are shown in Figure 3A. We identified 1106 proteins that were predicted to interact with RP4-694A7.2 (Figure 3A, $3 \mathrm{~B})$. Those proteins were mainly located in the nucleus (29.1\%) and cytosol (25.7\%) (Figure 3C). Next, a FISH assay was performed using SK-Hep1 cells to detect the location of RP4-694A7.2. Our results showed that RP4-694A7.2 was primarily located in the cytoplasm of SK-Hep1 cells (Figure 3D).

Based on these results, the functional enrichment analysis of 284 cytoplasmic proteins out of 1106 proteins was performed to further investigate their effects, which were mediated by highly expressed proteins associated with RP4-694A7.2, on physiological processes.

The functional protein activities were classified into three major groups by secondary GO analysis: cell component, molecular function, and biological process. Our results showed that cell part (276 proteins), organelle part (227 proteins), and organelle (197 proteins) were the top three cell components where proteins were located. The top three molecular functions were binding (270 proteins), catalytic activity (163 proteins), and molecular function regulation (66 proteins). Regarding biological processes, 264 proteins were related to cellular processes, 233 to biological regulation, and 204 to metabolic processes (Figure 3E).

The differentially expressed plasma proteins associated with RP4-694A7.2 were evaluated by KEGG analysis to analyze the major biological pathways and regulatory processes. The top 10 enrichment analysis pathways are shown in Figure $3 \mathrm{~F}$.

\section{Identification of proteins associated with RP4-694A7.2 in HCC cells}

To validate the results of proteomic analyses, we further selected 28 cytoplasmic proteins related to HCC for a PRM analysis. The screening criteria were selected based on the following principles: 1) proteins with potential biological function; 2) fold changes of $>1.2$ for upregulated proteins and $<0.8$ for downregulated proteins and a p-value of $<0.05$ were the established thresholds; and 3) inclusion in the top 10 enrichment analysis pathways.

PRM showed that 23 proteins were upregulated and 5 proteins were downregulated. The PRM results were consistent with those of TMT analysis (Figure $3 G)$. We further selected the proteins whose trend was 
consistent with that of RP4-694A7.2 for analysis. At last, five proteins-Annexin A4 (ANXA4), tyrosine 3-monooxygenase/tryptophan 5-monooxygenase activation protein theta (YWHAQ), tyrosine 3-monooxygenase/tryptophan 5-monooxygenase activation protein eta (YWHAH), PSAT1, and branched-chain amino acid transaminase 1 (BCAT1)-were included.

A

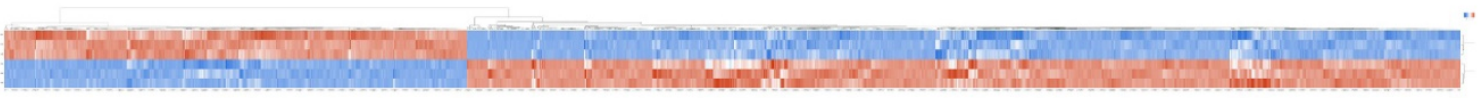

B

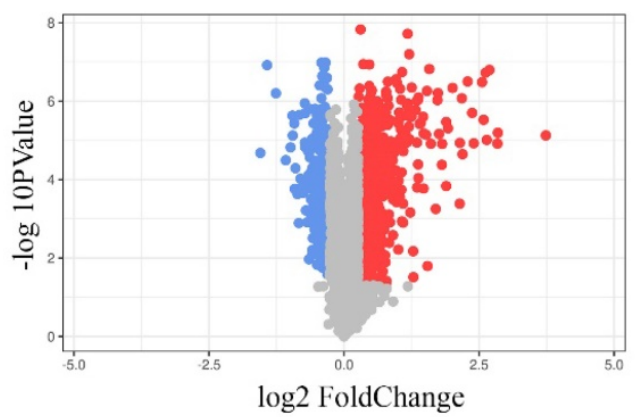

C

Location_Pie

- Down

- Unchange

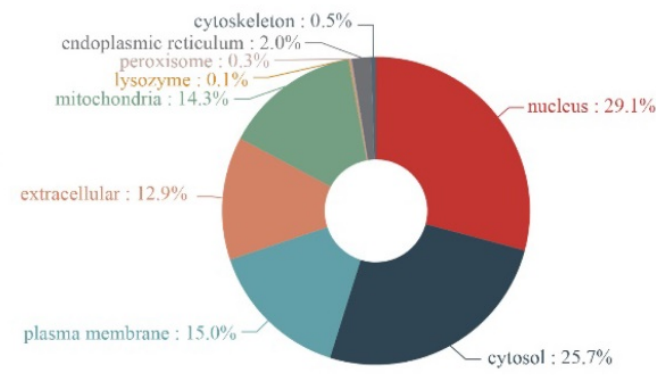

D

DAPI
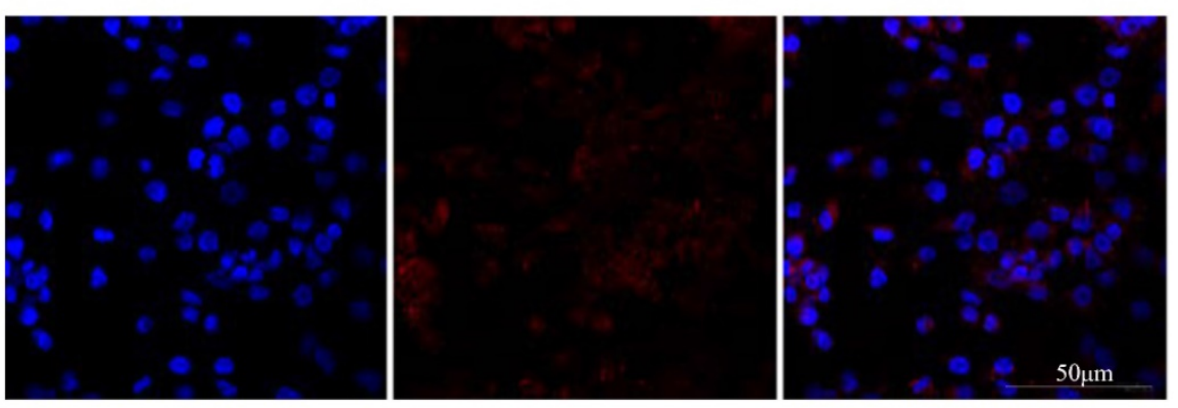

$\mathrm{E}$

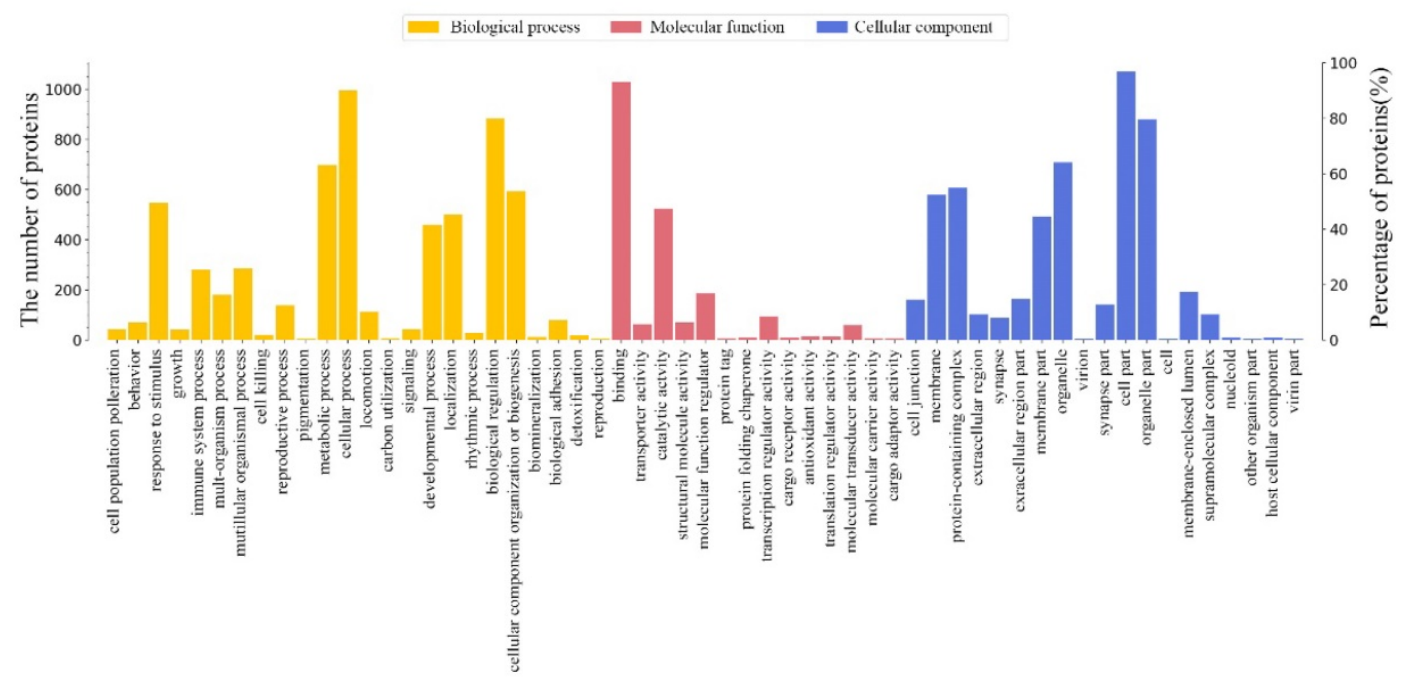


$\mathrm{F}$

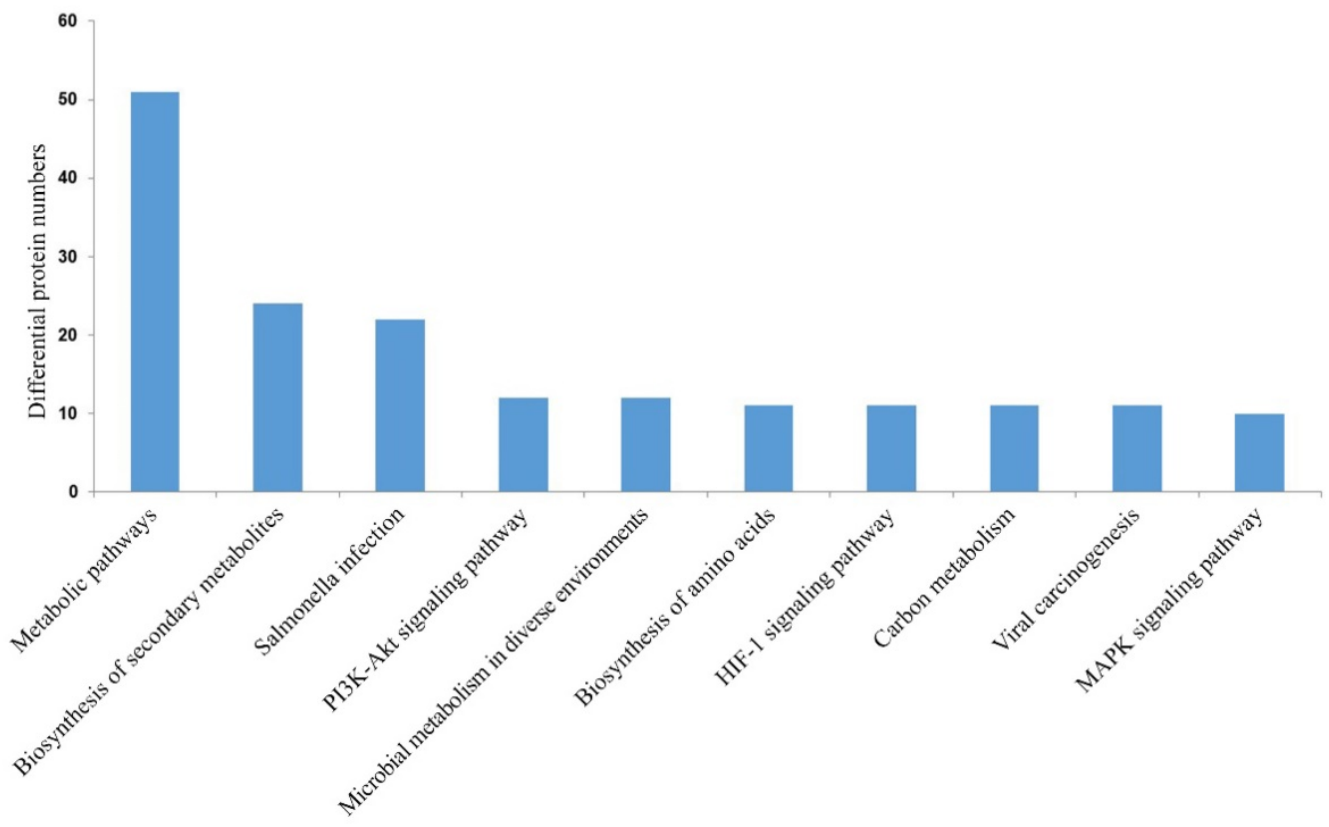

G

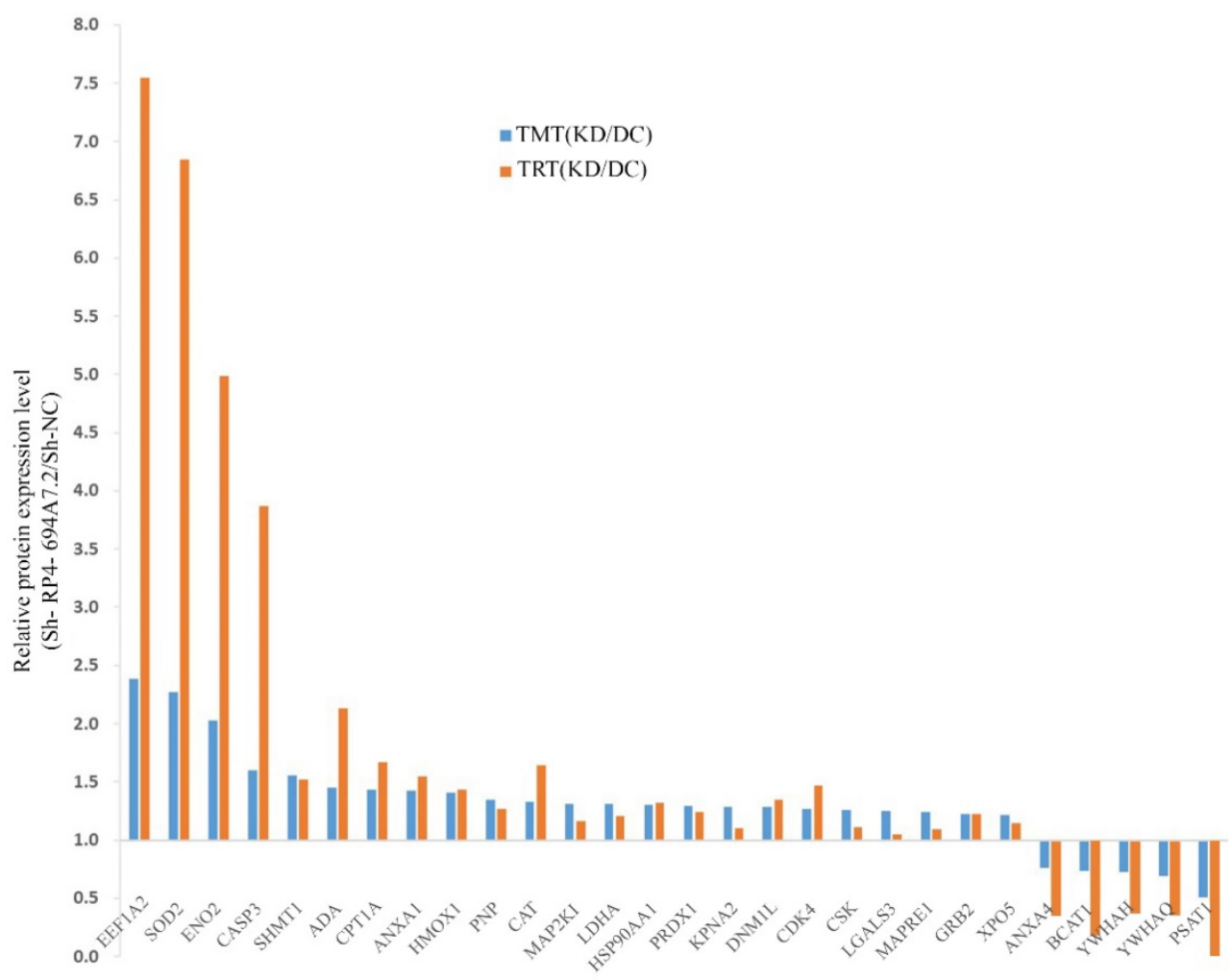

Figure 3. Screening and identification of differentially expressed proteins associated with RP4-694A7.2. A: Qualitative and quantitative analyses of proteomics data. Unsupervised hierarchical clustering of all proteome datasets showed separation among the different groups. Protein expression levels are colored, showing higher and lower expression in red and blue, respectively (TMT). B: Volcano map of differentially expressed proteins. C: Subcellular location of differentially expressed proteins. D: FISH assay of the distribution of RP4-694A7.2 expression in cells ( $\times 400$; red represents RP4-694A7.2 expression and blue represent DAPI nuclear staining). E: Gene ontology analysis. The differentially expressed proteins are mainly classified in terms of molecular function, cell composition, and biological process, respectively. F: Top 10 pathways of cytoplasmic proteins related to RP4-694A7.2, as determined by KEGG Pathway Analysis. G: Comparison of relative expression levels of 28 proteins validated by RPM in SK-Hep-1 cells. Results of the RPM analysis were consistent with TMT results. 


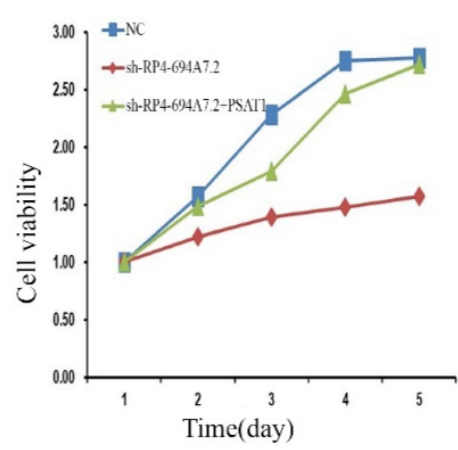

B sh-NC
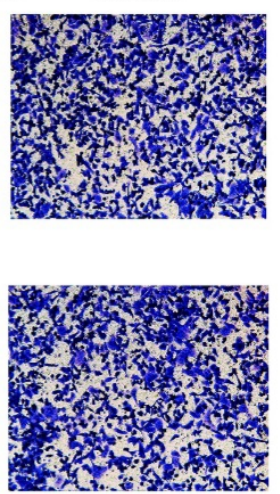

sh-RP4-694A7.2 sh-RP4-694A7.2+PSAT1
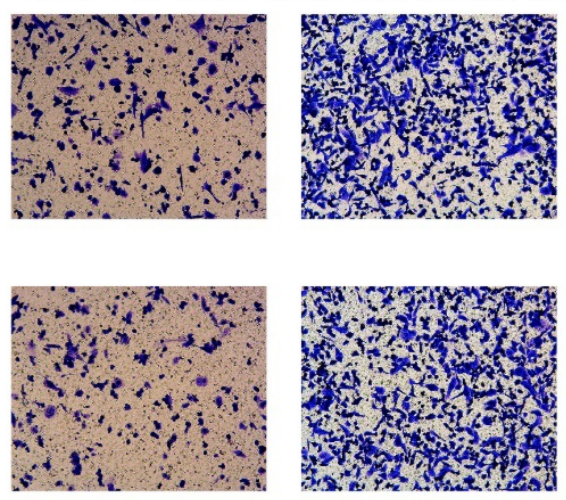

C
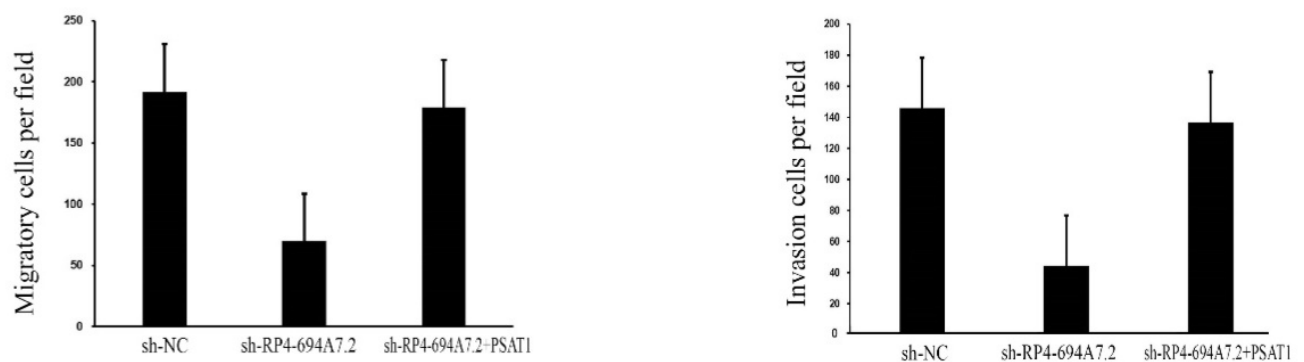

Figure 4. RP4-694A7.2 promoted cell proliferation, invasion, and migration by regulating PSAT1 in SK-Hep-1 cells. A: After SK-Hep-1 cells were transfected with sh-NC, sh-RP4-694A7.2, sh-RP4-694A7.2, and PSATI plasmids for 5 days, cell proliferation was examined by a CCK-8 assay ( $<0.05)$. B: After SK-Hep-1 cells were transfected with sh-NC, sh-RP4-694A7.2, sh-RP4-694A7.2, and PSAT1 plasmids for $72 \mathrm{~h}$, their migration and invasion were detected by a Transwell assay. C: Quantification of the number of migration and invasion SK-Hep-1 cells $(P<0.05)$.

\section{LV-RP4-694A7.2 regulates the progression of HCC by interacting with PSAT1}

To further determine whether those proteins (PSAT1, YWHAQ, YWHAH, BCAT1, and ANXA4) mediate the effects of RP4-694A7.2 on proliferation, migration, and invasion in $\mathrm{HCC}$, a phenotypic rescue assay was performed. The results showed that the proliferation of SK-Hep1 cells with RP4-694A7.2 knockdown was significantly reduced, while the phenomenon was partially reversed by PSAT1 and BCAT1 plasmids $(\mathrm{p}<0.05)$. Furthermore, the migration and invasion of SK-Hep1 cells with RP4-694A7.2 knockdown were significantly reduced, while the phenomenon was partially reversed by PSAT1, YWHAQ, YWHAH, and ANXA4 plasmids ( $\mathrm{p}$ $<0.05$ ).

These results showed that PSAT1 is necessary for the RP4-694A7.2-mediated regulation of HCC cell proliferation (Figure 4A), migration, and invasion (Figure 4B).

\section{Discussion}

HCC is a common malignant tumor and its progression is complex, involving cross-talk among many factors, including both genetic and epigenetic changes. Recently, IncRNAs have emerged as important contributors to the biological processes underlying the pathophysiology of many human diseases, including HCC [3]. A number of lncRNAs have been identified because of advances in bioinformatics approaches and next-generation sequencing technology. Networks composed of lncRNAs regulate proliferation, metabolism, metastasis, and other processes in various malignant diseases.

In this study, we first identified dysregulated lncRNAs in two GSE data sets related to HCC generated by high-throughput sequencing. We found that RP4-694A7.2 expression is higher in HCC malignant tissues than in normal liver tissues. Recently, RP4-694A7.2 has been found in some solid cancers [14,19]; however, its expression patterns and functions in HCC are still unknown. Our results suggested that RP4-694A7.2 is upregulated in HCC and might contribute to tumorigenesis.

Loss-of-function experiments further established the function of RP4-694A7.2 in vitro. The results of these analyses showed that RP4-694A7.2 promotes the proliferation, invasion, and metastasis in SK-Hep1 cells. These results showed that RP4-694A7.2 is an oncogene that promotes HCC development and metastasis. Our results that RP4-694A7.2 is related to increased tumor cell proliferation was consistent with those of a previously published study [19]. 
To explore the mechanism associated with the effects of RP4-694A7.2, FISH was used to determine the distribution of RP4-694A7.2 in SK-Hep1 cells. Next, TMT was performed to identify the proteins regulated by RP4-694A7.2. As RP4-694A7.2 was mainly distributed in the cytoplasm, 28 cytoplasmic proteins associated with HCC were detected by PRM analysis. Rescue experiments clearly indicated that PSAT1 was overexpressed in HCC and regulated cell proliferation and metastasis.

The serine synthesis pathway (SSP) is an important pathway in cancer metabolism [20]. PSAT1, 3-phosphoglycerate dehydrogenase (PHGDH), and phosphoserine phosphatase (PSPH) are three important regulators in the SSP. A study has found that the expression of PSAT1, which is an enzyme that catalyzes the second step of the SSP, is related to cell proliferation and cancer development [21]. High PSAT1 expression has been detected in non-small cell lung cancer, breast cancer, and esophageal squamous cell carcinoma, and PSAT1 expression can enhance tumorigenesis and metastasis [22-24]. Moreover, PSAT1 could be regarded as a prognostic marker in cancer, as PSAT1 overexpression could indicate a poor prognostic outcome [25]. Our results also indicated that PSAT1 is regulated by $\operatorname{lncRNA}$ RP4-694A7.2 to modulate HCC cell growth and metastasis.

RP4-694A7.2 has been proven to play an oncogenic role in the cancer development and pathogenesis; it may participate in cell-cycle control, and its silencing reduced the S-phase fraction together with a block of the G1/S phase [19]. Our results further showed that numerous proteins that interact with RP4-694A7.2 are associated with catalytic activity and molecular function regulation. PSAT1, a necessary enzyme that makes 3-phosphoglycerate from glycolysis and converts it to serine in the SSP [26], could promote cyclin D1 expression via the GSK3 $\beta / \beta$-catenin pathway, which eventually led to the acceleration of cell cycle progression [22]. In addition, a previous study showed that PSAT1 may participate in glycolysis and amino acid metabolism in human HCC tissue-derived metabolic [27]. Consistent with our PRM and TMT results, metabolic pathways were the most enriched. In summary, we speculated that RP4-694A7.2 may regulate glycolysis function by PSAT1 via the GSK3 $\beta / \beta$-catenin pathway during HCC cell growth and metastasis.

This study also has several limitations. Although we identified that RP4 694A7.2 may act as an oncogene to promote cell proliferation, invasion, and migration by targeting PSAT1, more regulatory factors and molecular elements that interact are still unknown. Further studies are required to clarify these molecular elements and the interactions among them. In addition, the clinical application of RP4 694A7.2 needs to be explored in the future.

In summary, the results of our study illustrate that RP4-694A7.2 is upregulated in HCC and promotes cell proliferation and metastasis via PSAT1.

\section{Acknowledgements}

This project was supported by the "Thirteenth Five-Year Plan" Major National Science and Technology Major Project (No. 2017ZX10201201, 2017ZX10202202) and the Liaoning Provincial Local Professional Technology Innovation Platform Construction Project (No. 2016007013).

\section{Competing Interests}

The authors have declared that no competing interest exists.

\section{References}

1. Global Burden of Disease Cancer C, Fitzmaurice C, Abate D, Abbasi N, Abbastabar H, Abd-Allah F, Abdel-Rahman O, Abdelalim A, Abdoli A, Abdollahpour I, Abdulle ASM, Abebe ND, Abraha H N, et al. Global, Regional, and National Cancer Incidence, Mortality, Years of Life Lost, Years Lived With Disability, and Disability-Adjusted Life-Years for 29 Cancer Groups, 1990 to 2017: A Systematic Analysis for the Global Burden of Disease Study. JAMA Oncol. 2019;5:1749-68.

2. Yang JD, Hainaut P, Gores GJ, Amadou A, Plymoth A, Roberts LR. A global view of hepatocellular carcinoma: trends, risk, prevention and management. Nat Rev Gastroenterol Hepatol. 2019;16:589-604.

3. Kim YA, Park KK, Lee SJ. LncRNAs Act as a Link between Chronic Liver Disease and Hepatocellular Carcinoma. Int J Mol Sci. 2020;21.

4. St Laurent G, Wahlestedt C, Kapranov P. The Landscape of long noncoding RNA classification. Trends Genet. 2015;31:239-51.

5. Kong S, Tao M, Shen X, Ju S. Translatable circRNAs and lncRNAs: Driving mechanisms and functions of their translation products. Cancer Lett. 2020;483:59-65

6. Cech TR, Steitz JA. The noncoding RNA revolution-trashing old rules to forge new ones. Cell. 2014;157:77-94.

7. Niu ZS, Wang WH, Dong XN, Tian LM. Role of long noncoding RNA-mediated competing endogenous RNA regulatory network in hepatocellular carcinoma. World J Gastroenterol. 2020;26:4240-60.

8. Qin G, Tu X, Li H, Cao P, Chen X, Song J, Han H, Li Y, Guo B, Yang L, Yan P, Li P, Gao C, et al. Long Noncoding RNA p53-Stabilizing and Activating RNA Promotes p53 Signaling by Inhibiting Heterogeneous Nuclear Ribonucleoprotein K deSUMOylation and Suppresses Hepatocellular Carcinoma. Hepatology. 2020;71:112-29.

9. Wang CZ, Yan GX, Dong DS, Xin H, Liu ZY. LncRNA-ATB promotes autophagy by activating Yes-associated protein and inducing autophagy-related protein 5 expression in hepatocellular carcinoma. World Journal of Gastroenterology. 2019;25:5310-22

10. Zhang H, Chu K, Zheng C, Ren L, Tian R. Pseudogene DUXAP8 Promotes Cell Proliferation and Migration of Hepatocellular Carcinoma by Sponging MiR-490-5p to Induce BUB1 Expression. Front Genet. 2020;11:666.

11. Huang JF, Jiang HY, Cai H, Liu Y, Zhu YQ, Lin SS, Hu TT, Wang TT, Yang WJ, Xiao B, Sun SH, Ma LY, Yin HR, et al. Genome-wide screening identifies oncofetal IncRNA Ptn-dt promoting the proliferation of hepatocellular carcinoma cells by regulating the Ptn receptor. Oncogene. 2019;38:3428-45.

12. Yang F, Jiang Y, Lv LZ. Long non-coding RNA XLOC_010235 correlates with poor prognosis and promotes tumorigenesis of hepatocellular carcinoma. Eur Rev Med Pharmacol Sci. 2017;21:4867-74.

13. Gu JX, Zhang X, Miao RC, Xiang XH, Fu YN, Zhang JY, Liu C, Qu K. Six-long non-coding RNA signature predicts recurrence-free survival in hepatocellular carcinoma. World J Gastroenterol. 2019:25:220-32.

14. Yan X, Hu Z, Feng Y, Hu X, Yuan J, Zhao SD, Zhang Y, Yang L, Shan W, He Q, Fan L, Kandalaft L E, Tanyi JL, et al. Comprehensive Genomic Characterization of Long Non-coding RNAs across Human Cancers. Cancer Cell. 2015;28:529-40.

15. Petri A, Dybkaer K, Bogsted M, Thrue CA, Hagedorn PH, Schmitz A, Bodker JS, Johnsen HE, Kauppinen S. Long Noncoding RNA Expression during Human B-Cell Development. PLoS One. 2015;10:e138236.

16. Edgar R, Domrachev M, Lash AE. Gene Expression Omnibus: NCBI gene expression and hybridization array data repository. Nucleic Acids Res. 2002;30:207-10. 
17. Elias JE, Gygi SP. Target-decoy search strategy for increased confidence in large-scale protein identifications by mass spectrometry. Nat Methods. 2007;4:207-14.

18. Cao C, Sun J, Zhang D, Guo X, Xie L, Li X, Wu D, Liu L. The Long Intergenic Noncoding RNA UFC1, a Target of MicroRNA 34a, Interacts With the mRNA Stabilizing Protein HuR to Increase Levels of $\beta$-Catenin in HCC Cells. Gastroenterology. 2015;148:415-26.e18.

19. Roisman A, Castellano G, Navarro A, Gonzalez-Farre B, Perez-Galan P, Esteve-Codina A, Dabad M, Heath S, Gut M, Bosio M, Bellot P, Salembier P, Oliveras A, et al. Differential expression of long non-coding RNAs are related to proliferation and histological diversity in follicular lymphomas. $\mathrm{Br} \mathrm{J}$ Haematol. 2019;184:373-83.

20. Huang SP, Chan YC, Huang SY, Lin YF. Overexpression of PSAT1 Gene is a Favorable Prognostic Marker in Lower-Grade Gliomas and Predicts a Favorable Outcome in Patients with IDH1 Mutations and Chromosome 1p19q Codeletion. Cancers. 2019;12.

21. Baek JY, Jun DY, Taub D, Kim YH. Characterization of human phosphoserine aminotransferase involved in the phosphorylated pathway of l-serine biosynthesis. Biochemical Journal. 2003;373:191-200.

22. Gao S, Ge A, Xu S, You Z, Ning S, Zhao Y, Pang D. PSAT1 is regulated by ATF 4 and enhances cell proliferation via the GSK3 $\beta / \beta$-catenin/cyclin D1 signaling pathway in ER-negative breast cancer. Journal of Experimental \& Clinical Cancer Research. 2017;36.

23. Chan $Y C$, Chang $Y C$, Chuang $H H$, Yang $Y C$, Lin $Y F$, Huang MS, Hsiao M, Yang CJ, Hua KT. Overexpression of PSAT1 promotes metastasis of lung adenocarcinoma by suppressing the IRF1-IFNY axis. Oncogene. 2020;39:2509-22.

24. Yang Y, Wu J, Cai J, He Z, Yuan J, Zhu X, Li Y, Li M, Guan H. PSAT1 regulates cyclin D1 degradation and sustains proliferation of non-small cell lung cancer cells. International Journal of Cancer. 2015;136:E39-E50.

25. Liu B, Jia Y, Cao Y, Wu S, Jiang H, Sun X, Ma J, Yin X, Mao A, Shang M. Overexpression of Phosphoserine Aminotransferase 1 (PSAT1) Predicts Poor Prognosis and Associates with Tumor Progression in Human Esophageal Squamous Cell Carcinoma. Cell Physiol Biochem. 2016;39:395-406.

26. Antonov A, Agostini M, Morello M, Minieri M, Melino G, Amelio I. Bioinformatics analysis of the serine and glycine pathway in cancer cells. Oncotarget. 2014;5:11004-13.

27. Nwosu ZC, Battello N, Rothley M, Pioronska W, Sitek B, Ebert MP, Hofmann U, Sleeman J, Wolfl S, Meyer C, Megger DA, Dooley S. Liver cancer cell lines distinctly mimic the metabolic gene expression pattern of the corresponding human tumours. J Exp Clin Cancer Res. 2018;37:211. 